\title{
Infants Born to HIV-1 Infected Women : Lessons from the Past Decade
}

\author{
Aditya Kaul and Sulachni Chandwani \\ Department of Pediatrics, Division of Infectious Diseases and Immunology. New York \\ University Medical Center, New York, USA
}

In 1981, what began as a small outbreak in white males in North America and Western Europe has rapidly evolved into a global pandemic, as the 20th century comes to an end. Estimates from WHO suggest that over 13 million people will be infected worldwide with the Human Immunodeficiency Virus Type 1 (HIV-1); this includes 3 million women, mostly of reproductive age and over a half million children. Over $80 \%$ of HIV infected people live in Africa, however, in recent years the epidemic has begun to move to Asia where the majority of the world's population lives. Over a million people are believed to be infected with HIV-1 in India and about half million in Thailand. The rapidity of its spread has coincided with a shift in the predominant mode of transmission to heterosexual route and therefore, into the general population. Based on World Heath Organization (WHO) projections, by the year 2000 , between 30-40 million people will be infected worldwide, of whom 3 million women and 2.7 million children will have died, and an additional 5.5 million will be orphaned. This epidemic may not peak in

Reprint requests: Dr. Aditya Kaul, Clinical Associate Professor of Pediatrics, Department of Pediatrics, Division of Infectious Diseases and Immunology, New York University Medical Center, 550 First Avenue, New York, NY 10016, USA.
Asia until the year 2015, reversing all the gains made in infant and childhood mortality in the last 2-3 decades. ${ }^{1-2}$

The first reports of AIDS in children began to appear in $1982-83 .^{3}$ The early years of the epidemic began with studies of epidemiology, risk factors and then the natural history of the infection in children. Vertical transmission of AIDS as an important link in establishing the viral etiology of AIDS. The emphasis gradually changed to studies of transmission rates, seroprevalence in pregnant women, and immunology of HIV-1, especially how it relates to the developing immune system. More recently, early diagnosis, treatment and prevention of opportunistic infections and in the late 1980's, specific antiretroviral therapies have been the subjects of research efforts.

\section{Vertical Transmission}

The rates of transmission on HIV from mother to infant have varied from 13 to $50 \%$. Prospective studies from Western Europe and North America have found transmission rates to vary from $13-32 \%$, whereas those from Africa and Haiti have found rates as high as $27-50 \%$. The reasons for these differences remain unclear at this time. Perhaps differences in maternal immune status, stage of disease or 
presence of other infections, such as genital ulcers may account for the higher transmission rates in Africa. ${ }^{5}$

Transmission of HIV-1 infection from mother to infant can occur in utero, during delivery, or in the post-partum period via breast milk. Detection of HIV-1 from fetuses between 9-20 weeks of gestation suggests that prenatal transmission does occur. ${ }^{4}$ Studies using newborn blood samples to detect HIV-1 by viral culture of HIV DNA by Polymerase Chain Reaction (PCR) find that only on half of all infected children have detectable virus. Based on these observations, it is believed that a large number of infants acquire HIV infection during the intrapartum period. Material characteristics that increase the risk of transmission include advanced HIV disease with low CD4 (T-helper) lymphocyte counts, primary infection during pregnancy, and clinical illness at the time of delivery. Preterm delivery $(<34$ weeks) may result in an increase in the rate of transmission.

Postnatal transmission of HIV-1 through breastfeeding was first reported in infants whose mothers acquired HIV-1 infection following transfusion in the postpartum period. Several reports subsequently have confirmed these earlier findings. HIV-1 has been demonstrated in the cellular and cell free fractions of breast milk. A meta-analysis of several studies estimated a $14 \%$ incremental risk of HIV-1 transmission in breast fed infants born to HIV infected mothers. ${ }^{6}$ As a results, in the U.S. HIV infected women are advised against breast feeding. Nonetheless, because of the benefits of breastfeeding in preventing malnutrition and in lowering infant mortality, WHO recommends breast-feeding as the best option for most regions of the world. ${ }^{7}$ Analysis of data from several observational studies from Europe and Nurth America shows that Caesarian section may result in a small. albeit significant reduction in the rate of HIV-1 transmission fro mother to fetus. Many issues, including the increased cost of Caesarian sections, need to be carefully reviewed before any firm recommendations can be made about the preferred mode of delivery.

\section{Care of HIV-1 Infected Women and their Children}

In response to the challenge of AIDS in New York, a comprehensive management program has been developed at Bellevue Hospital/NYU Medical Center over the last 10-12 years. Pregnant women with HIV-1 infection are identified early in pregnancy and enrolled into a high risk obstetric programme. Postnatally, these mothers and their infants are followed by a team comprised of pediatric and adult infectious diseases specialist, obstetricians pediatric neurokogists, psychologists, dentists, nurses, social workers, and other support staff. The major goals of this program are, provison of clinical services, enrollment of subjects into various treatment trials and research studies focusing on the immunopathogenesis of HIV-1 infection and the role of associated opportunistic pathogens in the HIV disease progression. During the last decade, over $250 \mathrm{HIV}$ infected children and an additional 300 seroreverting children born to HIV infected mother have been followed at this center. The seroreverting children, who often retain maternal antibody for over a year before becoming antibody negative, are seen 
freguently for at least the first 2 years of life and often well beyond that age.

\section{Care of HIV-1 Infected Pregnant Women}

During the last 6-8 years, as evidence by anonymous testing of heelstick blood samples of newborns by the N.Y. State laboratories, the seroprevalence of HIV-1 infection in the obstetric population at $B H C$, has varied between 2-3\%. Serologic testing for HIV is voluntary in NY state, therefore intravenous drug using women or women engaging in sex with multiple partners are identified, counseled and tested only if they consent to testing. Immunologic function is monitored in these women. A significant non-reversible decline in the number of CD 4 (T-helper) lymphocytes can occur in HIV infected women, placing them at risk of developing opportunistic infection. Although no adverse effects of $Z D V$ on the developing fetus have been noted so far, the safety of antiretrovirals, particularly in the first trimester remains unclear at this time. Recently, a collaborative study from North America and Europe provided perhaps the most important finding regarding vertical transmission of HIV. It showed that Zidovudine, when given to pregnant women during the third trimester and the intrapartum period, and then to the newborn during the first 6 weeks of life, result in a $67.5 \%$ reduction in the rate of transmission of HIV-1 from mother to infant. ${ }^{9}$ This has resulted in revised recommendations for the use of antiretrovirals in HIV-1 infected pregnant women.

Other significant issues in the care of HIV infected women include screening and treatment for STD's like syphilis and gonorrhea and for HIV related infections, in particular, tuberculosis and Pneumocystis carinii. Procedures like am niocentesis, chorionic villous sampling and fetal scalp electrode monitoring expose the fetus to maternal blood and perhaps increase the risk of HIV transmission. Exposure to HIV infected blood and other secretions during labor and delivery mandates that health care workers use universal precautions which include gloves, gowns, masks, eyeshield, etc.

\section{Care of the Newborn}

At birth, infants who are ultimately proven to be HIV infected are clinically indistinguishable from those infants who do not acquire infection. In the U.S., the rates of perinatal complications, such as stillbirth, preterm delivery, and low birth weight do not seem to be more common in infants born to HIV infected women. This may not necessarily be the case in other countries, particularly in Africa and Asia.

There are several specific concerns in the care of infants born to HIV infected mother during the immediate newborn period and thereafter. Routine newborn care, including screening and therapy of vertically acquired infection i.e. syphilis, herpes simplex, and rubella, immunization for Hepatitis B is given. These infants are usually discharged within the first week of life and followed as outpatients.

\section{Early Diagnosis of HIV Infection}

Early and accurate diagnosis of HIV infection in the infant is perhaps the most crucial question following birth to an HIV infected mother. The traditional serologic tests to detect HIV-1 antibody, Enzyme linked immunoassay (ELISA) and western blot, cannot identify infected infants reliably until they are 15-18 months of age, 
because of transplacental passage of maternal; antibodies. Aciditionally $50^{\circ}$ of uninfected infants test positive fo antibodies to HIY by EISSA at 1 vear of age. Meanwhile mani infezted infants may die of preventable causes Pneumocystis carinii) before a specific diagnosis of HIV infection is made.

Several techniques including viral culture, detection of viral DNA by polymerase Chain Reaction (PCRi and plasma
224 antigen assav have been utilized to de tect HN during infancy. In our laborator: an accurate diagnosis of HIV infection can be made in $50 \%$ of infected infants from blood sanples obtained in the first 2 dars of life and over $90^{\circ}$. by 2 months, using culture and PCR. ${ }^{10} \mathrm{HIV}$ p 24 antigen (unbound assay is less sensitive and more specific in the first 2 months of life, thereat. ter, the sensitivities of the p24 assay afproach those seen with culture and $P C R$.

Table 1. Age Related Frequency of Various Clinical Features and Secondary Infections in HIV:Infected Children"

\begin{tabular}{|c|c|c|}
\hline & \multicolumn{2}{|c|}{ Age } \\
\hline & $0-2$ yr & $>2 \mathrm{yT}$ \\
\hline I iepatosplenomegaly & +++ & ++ \\
\hline Generalized lymphadenopathy & t++ & t+ \\
\hline Growth failure & +++ & ++ \\
\hline Parotitis & \pm & ++ \\
\hline Lymphoid interstitial pneumonitis & \pm & +- \\
\hline Progressive encephalopathy & t+ & ++ \\
\hline Cardiomyopathy & + & + \\
\hline Thrombocrtopenia & $t^{++}$ & +- \\
\hline Nephropathy & $\cdot-$ & - \\
\hline Sinopulmonary infections & +++ & $+\div+$ \\
\hline Frequent otitis media & +++ & ++ \\
\hline Bacteremia & +++ & + \\
\hline Candidiasis & +++ & ++ \\
\hline Pneumocystis carinii infection & +++ & + \\
\hline Cytomegalovirus infection & ++ & + \\
\hline Zoster infection & - & ++ \\
\hline Atypical mycobacterial infection & \pm & ++ \\
\hline Herpes simplex infection & \pm & + \\
\hline
\end{tabular}

+++ very common, ++ common, + occasional, $=$ rare, - not seen

- Based on observation in over 250 children seen at Bellevue Hosp Ctr. Incidence of tuberculosis, cryptococcus, cryptosporidium, measles, toxoplasmosis and neoplasms was $<1-2^{\prime \prime}$, (each) of population. 
The sensitivity of 24 antigen assay can be improved by immune complex disosia tom. "Other methods used for early diag

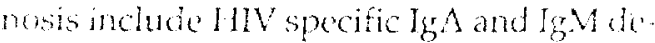
toction, in viro antibody production and the Elispot assay. Many of these techniques are expensive and not readily available and in the case of HIV culture, require 2-4 weeks. Inexpensive and reliable tests, available in kit form for use in the clinical laboratory are surely needed and will greatly facilitate the management of HIV infected infants. ${ }^{12}$

\section{Patterns of Clinical Disease}

HIV infected infants and children develop a variety of signs and symptoms which include, non-specific findings such as hepatosplenomegaly, diffuse lymphadenopathy, growth failure, parotitis, etc., or HIV related diseases like encephalopathy, cardiomyopathy, hepatitis, lymphoid pneumonitis, nephropathy, etc., and secondary bacterial, viral, protozoal or fungal infections. ${ }^{13,14}$ These disease processes occur at varying intervals after birth. A bimodal disease pattern is now well recognized, consisting of a rapidly progressive early onset form with lymphadenopathy, hepatosplenomegaly, oropharyngeal through by 3 to 4 months of age, followed by pneumocystis pneumonia (PCP) and encephalopathy. These infants show rapid and progressive clinical and immunologic deterioration and rarely survive beyond 45 years. In the slowly progressive form, children may present with non-specific findings early or may remain asymptomatic for several years. ${ }^{15}$ In our own patient population, $18 \%$ of children first presented with HIV related symptoms after 4 years of age. ${ }^{16}$

Recurrent sinopulmonary infection, bacteremia, especially due to sereptocor

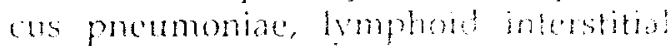

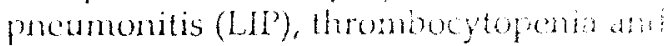
parotitis are some of the more common teatures of the slowly progressive discase. Table 1 summarizes the relative frequency of clinical findings in the early and late forms of disease seen at our institution during the past decade. Tuberculosis, al though quite common in HIV infected adults in New York city, has so far not been a problem in our patient population. In areas with a higher prevalence of tuberculosis in the general population, HIV infected children are at much greater risk of developing tuberculosis.

Growth failure is a common problem in HIV infected children, becoming evident as early as 4 months of age. Both weight and height are affected in these children, who appear younger than their chronologic age, rather than looking "wasted". Wasting syndrome, on the other hand is seen late in the course of HIV infection. The mechanism of growth failure remains unclear at present. In many children, a growth spurt may occur following institution of antiretroviral therapy. ${ }^{17}$

\section{Immunization of HIV Infected Children}

Recommendations for immunizing children born to HIV infected women differ in the U.S. from those made by WHO, and are summarized in Table 2. ${ }^{18,19}$ Live-viral of bacterial vaccines are not recommended for use in the U.S. Limited studies, however, of the MMR vaccine in both symptomatic and asymptomatic HIV infected children have not found any serious or unusual adverse events, hence, MMR vaccine is recommended for all children. Enhanced inactivated polio vaccine (eIPV) is preferred for use in the 
TABLE 2. Immunization of HIV-Infected Infants and Children, Recommendations of the ACIP and WHO (EPI)

\begin{tabular}{lcc}
\hline & ACIP & EPI \\
\hline DTP & Yes & Yes \\
OPV & No & Yes \\
eIPV & Y'es & - \\
MMR* & Yes & Yes \\
Hib & Yes & - \\
Hepatitis B** & Yes & - \\
Pneumococcal & Y'es & - \\
Influenza & Yes & - \\
BCG & No & Yes! \\
\hline
\end{tabular}

EPI schedule only includes single-agent measles vaccine; **Now recommended for all infants in the U.S.; !Not recommend for use in symptomatic subjects

U.S. Although a few studies suggest that B.C.G. may be safe in asymptomatic HIV infected children, it is not recommended for use in the U.S. The immune response to vaccines is generally suboptimal in symptomatic HIV infected children and adults and further decreases with progression of disease. Use of higher doses and frequent boosters have not been studies at present.

Immunologic Monitoring and Antiretroviral Therapy

The characteristics hallmark of HIV infection is a steady decline in the number of CD4 (T-helper) lymphocytes. Monitoring of CD4 lymphocyte numbers is an important component of long term care of these children. A progressive impairment of im- mune function, particularly, cellular immunity results in infections due to a variety of opportunistic pathogens. In the U.S. and Europe, Pneumocysits carinii, cytomegalovirus, varicella and herpes simplex viruses, candida and mycobacterium avium complex (MAC) along with Streptococcus pneumoniae are the most common pathogens. In Africa, other pathogens such as $M$. tuberculosis and cryptococcus may be more frequent.

The major goals of therapy, therefore, include drugs that block the replication of HIV in efforts to slow down the decline in cellular immunity. Secondly, prevention and treatment of secondary infections has resulted in most of the gains in survival made in the last decade. Specific antiretroviral agents, primarily nucleoside analogues such as azidothymidine (AZT), and dideoxyinosine (ddI) and dideoxycytidine ( $\mathrm{ddC}$ ) have been used for the last several years. Since the risk of opportunistic infections increases with declining numbers of CD4 lymphocyes, current guidelines for specific antiretroviral therapy and chemoprophylaxis for $P$. carinii are based upon the numbers of $\mathrm{CD} 4$ lymphocytes at different ages (Table 3 ). ${ }^{20}$ Chemoprophylaxis has also been used successfully for Candida, $M$. avium and $M$. tuberculosis infections in HIV infected adults.

Use of specific antiretroviral agents and prevention and treatment of opportunistic infections, perhaps more important, has greatly improved survival and function in HIV infected children. Today it is not uncommon to see a 10 year old child, infected in the perinatal period, who continues to lead a near normal life at home and in school. 
Table 3, Guidelines for Initiation of Antiretroviral. Therapy and Pneumocystic carinii (PCP) Prophylaxis Based on Either Number of Percentage of CD4 Lymphocytes*

\begin{tabular}{lcc}
\hline Age & \multicolumn{2}{c}{$\begin{array}{c}\text { CD4 lymphocyte } \\
\text { number (\%) }\end{array}$} \\
\cline { 2 - 3 } & $\begin{array}{c}\text { PCP } \\
\text { prophylaxis }\end{array}$ & $\begin{array}{c}\text { Antiretroviral } \\
\text { therapy }\end{array}$ \\
\hline$<12$ months & $1500(20 \%)$ & $1750(30 \%)$ \\
$12-24$ months & $750(20 \%)$ & $1000(25 \%)$ \\
$2-6$ years & $500(20 \%)$ & $750(20 \%)$ \\
26 years & 200 & 500 \\
\hline
\end{tabular}

*1. Adapted from reference \# 20;2. Patients with previous PCP should receive prophylaxis regardless of CD4 count or percentage; 3 . Children with AIDS defining illness should receive antiretroviral therapy regardless of CD4 count or percentage

\section{Issues for the Developing World}

Much of what has been learnt from studies in the U.S. and Europe is globally applicable in the management of HIV infected women and their children. However, many issues will have to resolve regionally or even individually by many nations. Principle among these are studies to estimate the prevalence of HIV infection in women of childbearing age. Estimates of vertical transmission rates also need to be made regionally before instituting preventive strategies.

Unlike the U.S. and Europe, P. carinii is not the most common opportunistic pathogen in Africa. Tuberculosis and Measles are much more frequent in African children and contribute significantly to their increased mortality rates. Hence, the natural history and frequency of various opportunistic pathogens need to be studied regionally in order to reduce the morbidity and mortality from these infections.

Currently available diagnostic tests, especially those used for early diagnosis of HIV infection in infancy continue to be very expensive and labor intensive. A defi. nite need exists for cheaper but sensitive and specific diagnostic test. Similarly, the cost of the presently available antiretroviral drugs may be prohibitively expensive for many populations, particularly in view of their limited efficacy. Use of Zidovudine in preventing vertical transmission may perhaps be the most cost effective strategy for many nations.

Much has been learned in the past decade, in particular, through the effects of the AIDS Clinical Trials Group (ACTG) formed by the National Institutes of $\mathrm{Al}$ lergy and Infectious Diseases, and by collaborative groups in Europe. Controlled clinical trials are being conducted for the treatment and prevention of HIV-1 and its relaked infections, using antiretrovirals and other drugs, biological agents and immunotherapies. Along with hope, however, have come many disappointments. We now realise that Zidovudine and other nucleoside analogues, alone and in combination with other antiretroviral agents provide only a limited benefits, delaying ultimate disease progression. Similarly, studies of candidate HIV vaccines have shown that antibodies in vaccine recipients can neutralize laboratory grown HIV-1 but not primary HIV-1 isolates from infected people.

A multipronged global effort must continue in order to combat the growing threat of AIDS and HIV infection. 


\section{References}

1. World Health Organization. The HIV/ AIDS Pandemic. 1993 Overview. Geneva : WHO/GPA/CNP/93 : 1 .

2. Chin J. Current and future dimensions of the HIV/AIDS pandemic in women and children. Lancet 1990, $144: 1210-1215$.

3. Rubinstein A, Sicklick M, Gupta A et al. Acquired immunodeficinecy with reversed $\mathrm{T} 4 / \mathrm{T} 8$ ratios in infants born to promiscuous and drug-addicted mothers. JAMA 1993; $249:$ : 2350-2356.

4. Oxtoby MJ. Vertically acquired HIV infection in the United States. In : Pizzo PA and Wilfert CM (eds). Pediatric AIDS : The challenge of HIV infection in infants, children, and adolescents. Baltimore, 2nd edition. Williams and Wilkins, 1994 : 320.

5. Ryder RW, Nsuami M, Nsa W et al. Mortality in HIV-1 seropositive women, their spouses and their newly born children during 36 months of follow up in Kinshasa, Zaire. AIDS 1994; $8: 667-672$.

6. Dunn DT, Newell ML, Ades AE, Peckham CS. Risk of human immunodeficiency virus type I transmission through breast-feeding. Lancet 1992; 340 : 585-588.

7. HIV transmission and breast-feeding. Bull World Health Organization. 1992; 70 : 667669.

8. The European Collaborative Study. Caesarean section and risk of vertical transmission of HIV-1 infection. Lancet 1994; 343 : 1464-1467.

9. CDCP. Zidovudine for the prevention of HIV transmission from mother to infant. MMWR 1994; 43 : 285-287.

10. Borkowsky W, Krasinski K, Pollack H et al. Early diagnosis of human immunodeficiency virus infection in children $<6$ months of age : Comparison of polymerase chain reaction, culture and plasma antigen capture techniques. J Infect Dis 1992; 166 : 616-619.

11. Chandwani S, Moore T, Kaul A et al.
Early diagnosis of human immunodeficiency virus type 1 infected infants by plasma p24 antigen assay after immune complex dissociation Pediatr Inf Dis $J$ 1993; 12 : 96-97.

12. Tamashiro $H$, Maskill $W$, Emmanuel J et al. Reducing the cost of HIV antibody testing. Lancet 1993; $342: 87-90$.

13. Krasinski $K$, Borkowsky $W$, and Holzman RS. Prognosis of human immunodeficiency virus infection in children and adolescent. Pediatr Inf Dis / 1989; 8 : 216220.

14. Tovo PA, DeMartino M, Gabiano $C$ et al. Prognostic factors and survival in children with perinatal HIV-1 infection. Lancet 1992; 339 : 1249-1253.

15. Blanche $S$, Tardieu M, Duliege AM et al. Longitudinal study of 94 symptomatic infants with perinatally acquired Human Immunodeficiency virus infection. $\mathrm{Am}$ / Dis Child 1990; 144 : 1210-1215.

16. Persaud $D, C$ handwani $S$, Rigaud $M$ et al. Delayed recognition of human immunodeficinecy virus infection in preadolescent children. Pediatrics 1992; 90 : 688-691.

17. McKinney RE, Robertson WR and the Duke Pediatric AIDS Clinical Trials Unit. Effect of human immunodeficiency virus infection on the growth of young children. J Pediatr 1993; 123 : 579-582.

18. CDCP. Recommendations of the Advisory Zommittee on Immunization Practices (ACIP) : Use of vaccines and immunoglobulins in persons with altered immunocompetence. MMWR 1993; 42 (No. RR-S) : 1-18.

19. World Health Organization. EPI for the 1990's. Geneva : WHO, March 1992.

20. El-Sadr W, Oleske JM, Agins BD et al. Evaluation and Management of Early HIV Infection. Clinical Practice Guideline No. 7. AHCPR Publication No. 94-0572 Rockville MD : Agency for Health Care Policy and Research, Public Health Service, U.S. Department of Health \& Human Services, Jan 1994. 